\title{
Design Consideration for Front-End System in Ultrasonic Tomography
}

\author{
Nor Muzakkir Nor Ayoba, Muhammad Jaysuman Pusppanathanª, Ruzairi Abdul Rahima ${ }^{*}$, Mohd Hafiz Fazalul Rahiman ${ }^{b}$, Fazlul Rahman \\ Mohd Yunus ${ }^{b}$, Salinda Buyamina, Intan Maisarah Abd Rahima, Yusri Md. Yunosa
}

\begin{abstract}
aProcess Tomography and Instrumentation Engineering Research Group (PROTOM-i), Infocomm Research Alliance, Faculty of Electrical Engineering, Universiti Teknologi Malaysia, 81310 UTM Johor Bahru, Johor, Malaysia

${ }^{b}$ Tomography Imaging Research Group, School of Mechatronic Engineering, Universiti Malaysia Perlis, 02600, Arau, Perlis, Malaysia
\end{abstract}

*Corresponding author: ruzairi@fke.utm.my

\section{Article history}

Received :30 April 2013

Received in revised form : 9 July 2013

Accepted :25 July 2013

Graphical abstract

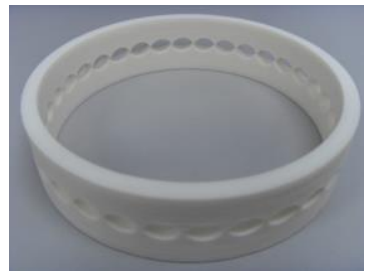

\section{Abstract}

The hardware development of the ultrasonic tomography comprises three main parts; the sensor unit, electronic measurement circuits combined with a data acquisition system and finally the display unit. The research done is focusing on the design considerations for the sensory unit which is also regarded as the front-end system. This part is an important concern for researchers in the field of ultrasonic, particularly for process tomography. Ultrasonic transducers are very sensitive piezo-mechanical component and as such its installation to complete the front-end system have to be properly configured. Many considerations and parameters that are well planned will promise significant impact on the sensor readings. Measurement errors can occur due to many aspects, thus this research aims to minimize such errors to be useful for further development in ultrasonic tomography system design.

Keywords: Front-end system; process tomography; sensor jig; ultrasonic transducer

(C) 2013 Penerbit UTM Press. All rights reserved.

\subsection{INTRODUCTION}

Ultrasonic sensing interaction between two-phase liquid and gaseous components is a complicated matter; however it is also the most common category of multiphase flows. Liquid and gas flows are probably the most important form of multiphase flow and it is found widely in industrial applications [1]. Thus it has attracted many scientists and engineers worldwide in developing novel techniques for this application as it is a challenging problem with important research values.

Monitoring flow state of two-phase flow is very important since flow pattern affects the stability of operation process and the measurement accuracy of other parameters [2]. Process tomography using ultrasonic sensing will rely upon detectable interactions both in a homogeneous transmission medium and from interfaces, for example at gas bubbles in a liquid. A variety of interactions may arise. The attenuation of incident waves due to the object or field of interest may be detected between a transmitter and a receiver. Variation in the speed of sound in an inhomogeneous material may be measured. Backscattering at interfaces may also be sensed. Thus the sensed interaction data may be used as source data for tomography $[3,16]$.

The interactions introduced above may be used to gain information relating to the internal nature of a process. Discerning the importance of successfully imaging the internal activities inside the investigated process column, much focus is needed to detect and monitor these interactions using the ultrasonic sensors. The front-end design consideration is much well noted to be prominent as it may provide raw data for further processing to yield tomographic results. The front-end system, also described as the sensory unit comprises of the ultrasonic transducers itself and the sensor's fixture jig. This paper discusses the front-end placement that is carefully done with reference on non-invasive fabrication technique.

\subsection{ULTRASONIC SENSOR}

Ultrasonic transducers are built around piezoelectric ceramic that vibrate at certain ultrasonic frequencies when a voltage is applied, and if operated differently, it will instead generate voltage when its diaphragm vibrates. The piezo-electric ceramics can be packaged in a variety of housing, depending on how they are used. For instance, ultrasonic transducers used in field-service are commonly contact sensors, and are contoured to the surface to be inspected. These transducers have special wear and handling requirements of how they are used.

Ultrasonic transducers perform according to two main parameters: resolution and sensitivity. The resolution of a particular transducer is denoted by its ability to discern between two discontinuities that are on top of one another. A transducer with sufficient resolution will stop ringing, or vibrating, from the first discontinuity before receiving the echo from the second discontinuity. If the ceramic does not stop ringing before the second 
echo is received, the second echo is masked from the test system. The sensitivity of an ultrasonic transducer refers to the ability to detect small discontinuities. Reference gas tubes with different calculated diameter are used to gauge the sensitivity of a particular transducer.

\subsection{Sensor Selection}

The selection of ultrasonic transducer must be suitable to the application design where the transducer's projection should be in a wide angle. This is important for successful implementation of fanshaped beam projection technique which is to increase the received ultrasonic projection signals for each excited transmitter pulse.

Selecting the transducers is also vital as it will be used for the sensing area. The important criteria include choosing the compromised central frequency that can detect small gas bubbles without reducing much attenuated signal which could result in undetected signal at the receiver's end for the chosen vertical column's diameter. The frequency of the transducer is chosen based on the required sensitivity and depth of penetration. It should be noted that the higher the frequency, the better the sensitivity, but lower penetration depth.

The center frequency of the selected ultrasonic sensor should also be noted, especially for detecting small gas bubbles. In principle, the wavelength of the ultrasonic beam is directly linked to the resolving power of the sensor system where in a given material, increasing the frequency may be expected to increase the resolution of the system [3].

Previous researches [4], [5] and [6] have been focused on the use of $40 \mathrm{kHz}$ ultrasonic transducer with a divergent wide beam angle.

An ultrasonic sensor with active vibrating frequency of 40 $\mathrm{kHz}$ only has a resolution of $19 \mathrm{~mm}$ [7] therefore the gas hold-up size or the gas bubbles should be at least $19 \mathrm{~mm}$ in average or it could not be sensed by the ultrasonic sensor array. In many applications this is considered insufficient as gas bubbles with a size of $19 \mathrm{~mm}$ is already considered large [8], thus smaller gas bubbles will not be registered for an Ultrasonic Tomography system that utilizes $40 \mathrm{kHz}$ excitation frequency. To increase the resolution capability, ultrasonic sensor with a higher vibrating frequency is used in this research.

The availability of low cost ultrasonic sensor with higher frequency in small packaging has limited choices as there is a design limitation for such specification. Smaller sized sensor is recommended as this will increase the number of sensors that can be installed in the specified area of interest. The ultrasonic sensor used in this research is a custom-ordered sensor with a much higher frequency, operated at $333 \mathrm{kHz}$, with outer diameter of $9.3 \mathrm{~mm}$ and width $8.9 \mathrm{~mm}$. Table 1 shows the specification of the chosen sensor.

Table 1 Ultrasonic sensor specifications

\begin{tabular}{ll}
\hline Center Frequency $(F c)(\mathrm{kHz})$ & $335.0 \pm 10$ \\
Echo Sensitivity $(\mathrm{dB})$ & $-65 \mathrm{~dB} @ F c$ \\
Nominal Impedance at $F c(\mathrm{ohm})$ & 1000 \\
Bandwidth $(-6 \mathrm{~dB})$ & $30 \mathrm{kHz}$ min \\
& $50 \mathrm{Vp}-\mathrm{p}(2 \%$ Duty cycle tone \\
Maximum Driving Voltage & burst) \\
\hline
\end{tabular}

An experimental setup is configured to assess the sensor's usability in terms of sensor output reading and also the wide angle reception.

\subsection{Sensor Placement}

The ultrasonic transducers are the utmost important element in an ultrasonic tomography system as they are the "eyes and ears". Thus it is also an important requirement for the correct alignment of the sensors with respect to the sensing area. The transducer vibrating element needs to be aligned perpendicular to the circumference of the investigated column so that the ultrasound transmission is also correctly aligned with the corresponding receivers.

Measurement errors can occur due to misalignment between the excited transmitter and their corresponding receivers. If the transmitters are optimally aligned with the receivers, the received signals should have the maximum amplitude compared to the case if the transmitters were not in optimum alignment. Similarly, if the transmitted transducer transmits ultrasound energy by a few degrees of either side of the optimum position, then it should be expected to give a drop in the amplitude of the received signal.

Thus it is important for a sensor fixture jig is used to mount and fix the ultrasonic transducers properly around the column's circumference. The design of the sensor jig is carefully constructed to ensure the sensor can be precisely mounted in their corresponding locations. This will ensure that the installed ultrasonic transducers are able to produce maximum transmitted ultrasound energy with proper sensors mounting.
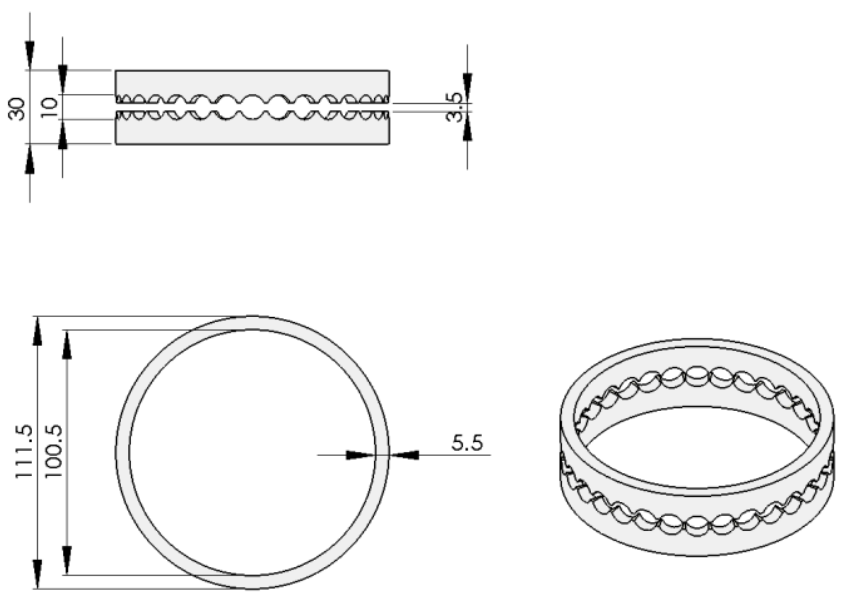

Figure 1 Sensor Fixture design (in millimeters)

To provide such support, the sensor fixture (Figure 1) used is manufactured using a 3D rapid prototyping printing (Figure 2) technique which uses Selective Laser Sintering (SLS) technique to produce a mechanical part of the fixture. This technique is used as it can provide the mechanical accuracy needed for optimum angle distribution of the sensor which cannot be provided by other methods such as CNC machining or manual mechanical manufacturing processes. 


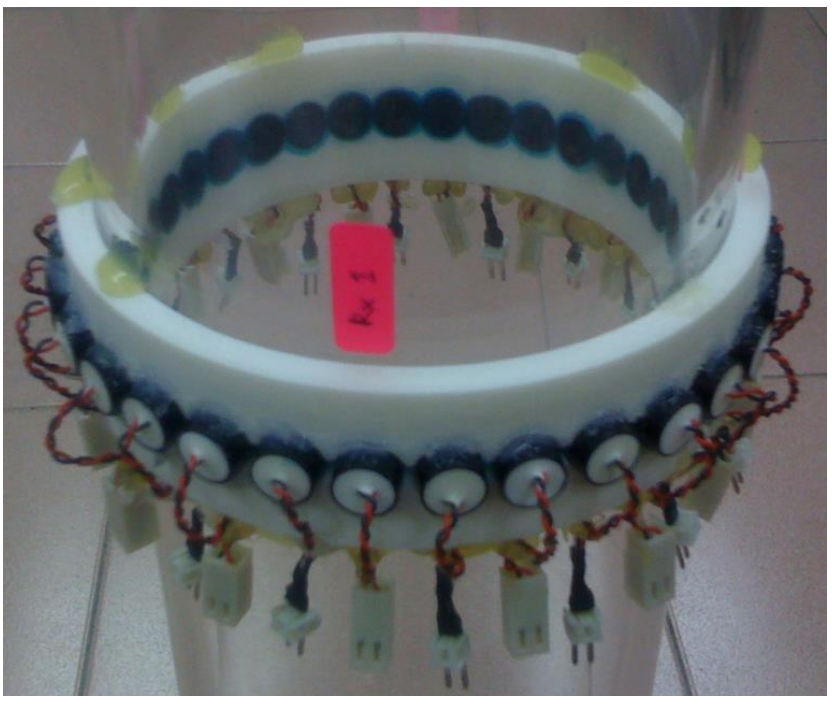

Figure 2 3D printed sensor fixture with installed ultrasonic sensors

\subsection{THE NON-INVASIVE TECHNIQUE}

During the past several years, the developers of non-intrusive and non-invasive techniques (Figure 3 ) have had a significant impact on our understanding of the fundamental hydrodynamics of multiphase industries. The recent progress in non-invasive techniques has been influenced largely by the development of the sensing techniques.

For many industrial applications it is desirable that the sensors are non-intrusive, i.e. they do not disturb the flow by extending into the process vessel, and non-invasive, i.e. they are not in direct contact with the flow medium. This can circumvent problems like leakage and corrosion while also operating the transducers in safer external configuration and prolonging the transducers' life.

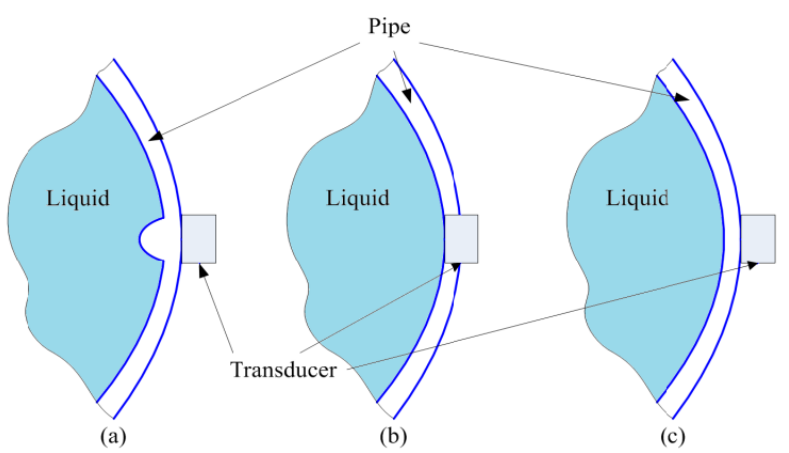

Figure 3 Invasiveness and intrusiveness of different sensor configurations

Non-invasiveness can be a very important requirement for industrial applications. There may be special demands in purity, like in food production, or the flow may contain aggressive and hazardous substances, like the chemical industries [9]. As shown in Figure 3(a), the non-invasive sensor element is not in direct contact with the flow. However, the configuration is intrusive as the pipe wall is formed to act as an acoustic lens spreading the sound beam.

Non-intrusiveness may also be of importance in order not to disturb the flow, leading to pressure drops and turbulences where such setup is depicted in Figure 3(b). The process column vessel contains slots through which the transducer elements are brought into direct contact with the flow medium. This approach avoids potential problems such as attenuation because of the pipe material, reflection loss due to acoustic mismatch and coupling between neighbouring transducers through structure-borne sound [9]. However, it is prone to problems like leakage and corrosion of the sensors.

The advantages of non-invasive instruments as in Figure 3(c) are obvious. They include reducing the hazards of operating with poisonous, radioactive, explosive, flammable or corrosive materials, minimizing problems with valuable process materials, avoiding contamination of pure or sterile materials, facilitating installations (and even retrofitting) and maintenance of the instruments even when the plant is on-stream. In an application requiring the use of a wide-angle projection beam of the ultrasonic transducers, this setup will of course require the selected sensors to have as wide as possible beam projection so that acoustic lens installation that could disturb the flow medium as in Figure 3(a) is not required.

Still, installing the ultrasonic sensors based on the noninvasive configuration as in Figure 3(c) however has a major disadvantage of creating a void at the boundaries between the transducer vibrating element and the vessel's wall. To maintain the penetration power of the ultrasonic wave's transmission, the void which is actually filled with air should be eliminated. This can be done by using viscous liquid to fill the void since the liquid will disperse to fill every space of a container/void, and maintains a fairly constant density. Ultrasonic wave at interfaces between the investigated mediums are very critical when using ultrasonic sensors for process tomography. This is mainly because the ultrasonic method in air is very inefficient due to mismatch of the sensor's impedance as compared with the air's acoustic impedance [10].

Successful implementation of an ultrasonic tomography system really relies on the sensing area, thus it cannot be stressed further about how important the sensors should be properly installed so that data can be acquired to produce a meaningful image. This is fundamental to the success or failure of an acoustic imaging system. Therefore, given the object to be imaged and the specifications to be achieved, the design of the front end of an acoustic imaging system should be regarded as a first priority [11].

The success of all acoustic imaging systems lies in matching the properties of the imaged objects with the related characteristics of ultrasound. In practice, if an ultrasonic transducer is placed against the surface of a material, very little ultrasonic energy will actually enter the material. This is because a very thin air layer will usually exist between the face of the transducer and the surface of the material. Air, being a very poor conductor of sound energy, will prevent effective coupling of the transducer to the material [12].

For this reason, a high-grade grease is used by the sensing area as the acoustic coupling to obviate gas components when mounting the ultrasonic transducers on the circumference of the pipe's wall as shown in Figure 4. Acoustic couplant placement will also significantly help to maximize the transmitted acoustic energy to the corresponding ultrasonic receivers. Proper installation and setup guidelines have been extensively explained in [13]. 


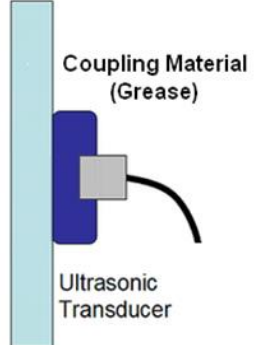

Figure 4 Acoustic coupling placement for ultrasound transmission

\subsection{RESULT AND DISCUSSION}

Based on the sensor arrangement as in Figure 5, a single transmitter is mounted in the $T x l$ position and another receiver is mounted based on the receivers' orientation placement. The transmitter is then excited with a minor frequency of $333 \mathrm{kHz}$ ultrasonic signal by producing two cycles of pulses from a microcontroller [7]; a major frequency of $1 \mathrm{kHz}$ is given before the transmitter is excited again repeatedly so that the reverberation effect is given time to fade off. The peak signal produced by the signal conditioning circuit connected to the receiver is observed and in turn the location of the receiver is rotated circularly from $R x l$ position until $R x l 6$.

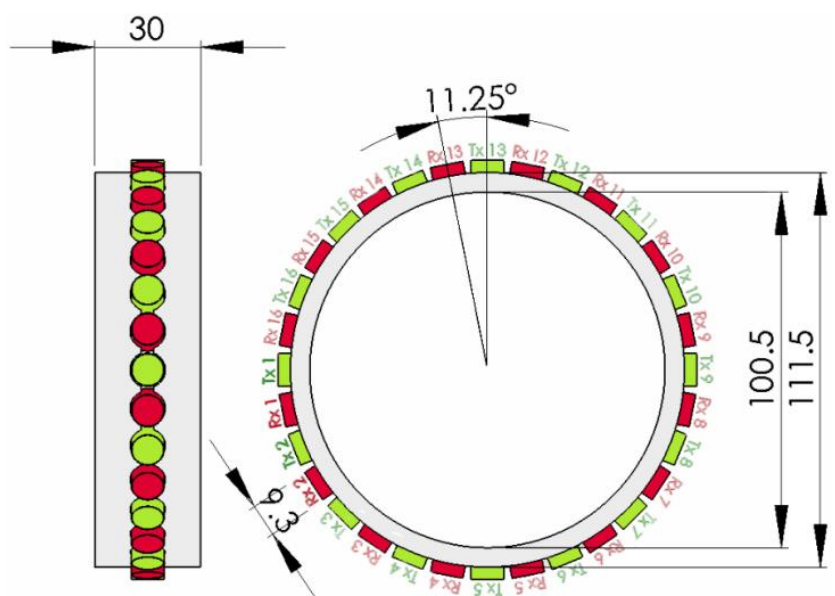

Figure 5 Sensor arrangement (in millimeters)

To determine the reception angle, the peak voltage is not the only parameter needed to be observed, the linear response of the received signals for different sensor locations is also monitored. This is done by inserting a gas-filled tube inside the vertical column (Figure 6) and the test tube is then waived in front of the projection path between the corresponding transmitter and receiver's location.

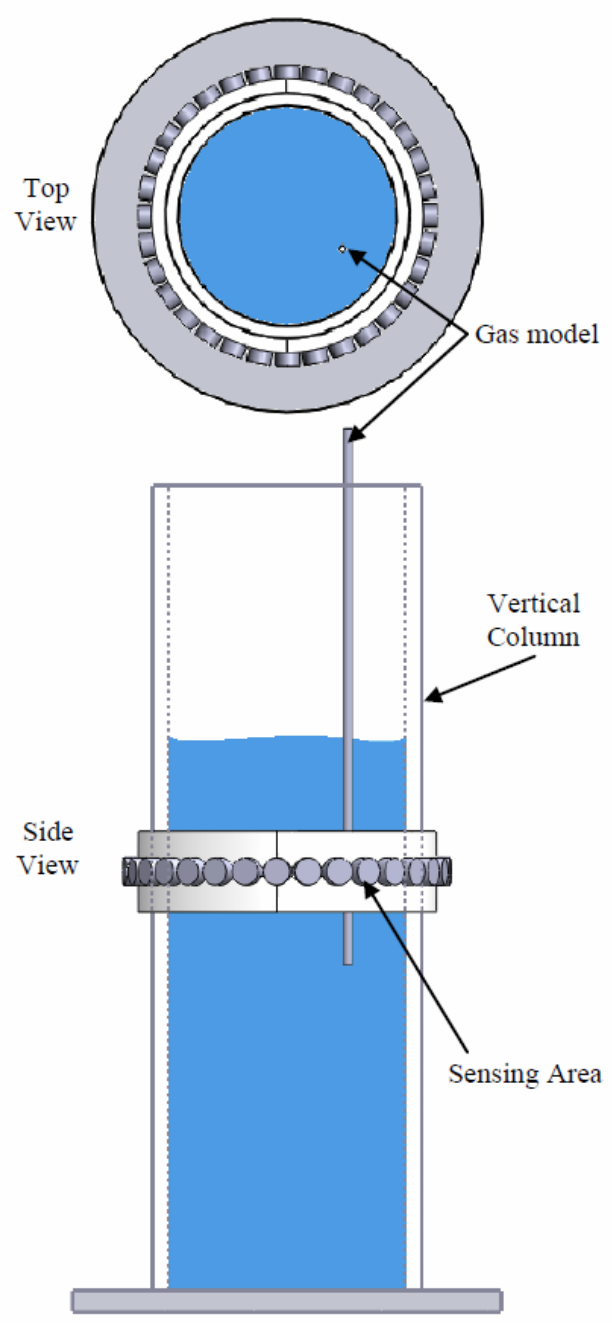

Figure 6 The experimental setup

At ultrasonic frequencies, there are material dependent losses that cause waves to attenuate as they propagate. Generally, the sources of the attenuation can be very complex [14]. Consider for example, a plane wave propagates through an attenuating medium as shown in Figure 7. The amplitude of this wave will change as it propagates.

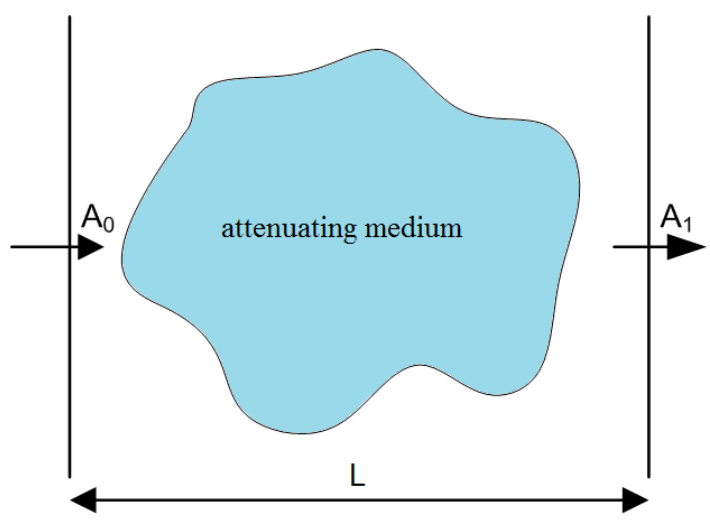

Figure 7 The ultrasonic attenuation model 
The effects of attenuation can be modelled by an exponential factor that contains a frequency dependent attenuation coefficient, $\alpha(f)$, distance of propagation in the medium $(L)$ and express the amplitude changes $\left(A_{1} / A_{0}\right)$ in this equation [14]:

$$
\frac{A_{1}}{A_{0}}=\exp [-\alpha(f) L]
$$

From the equation above, the attenuation will be critically dependent upon the material through which the ultrasound travels. An attenuation model for ultrasonic transducer based on transmission sensing mode can be further simplified as the Figure 8 .

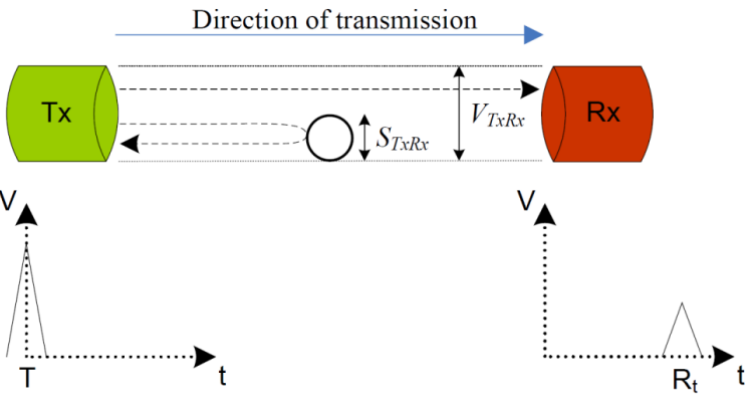

Figure 8 The attenuation model for ultrasound transmission

The ultrasonic receiver voltage (sensor value) is represented by $V_{T x, R x}$ and the sensor loss voltage due to the gas cavity is represented by $S_{T x, R x}$. The sensor loss voltage increases proportionally to the size of the gas cavity whereby the gas cavity blocks the ultrasound energy from being transmitted to the receiver. Therefore the receiver voltage (sensor value) is decreased as the sensor loss voltage increase. The peak voltage will have a high value when there's no gas obstruction along the projection path (straight-line assumption) and if vice versa, the voltage will have a lower value. The results obtained by using the ultrasonic sensor are graphically shown Figure 9.

\section{Voltage readings during reception angle observation}

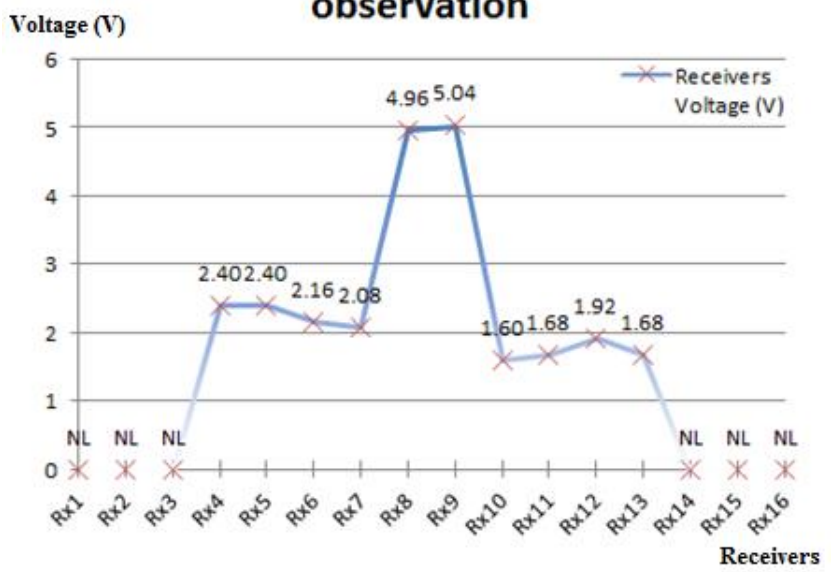

Figure 9 Ultrasonic receivers' voltage reading (NL - No Linearity)

From Figure 9, it is found that the sensor has a wide transmission angle where good linear response is received at 10 different sensor locations ( $\mathrm{Rx} 4-\mathrm{Rx} 13)$. This satisfies the characteristic needed to employ fan-shaped beam projection technique into the system and as thus qualifies the sensor for use in the sensing area.

\subsection{CONCLUSION}

The non-invasive fabrication technique has been introduced. In order to develop a successful non-invasive measurement section, acoustic coupling has been implemented between the transducer and the pipe wall. In this system, for the sensing area, a $333 \mathrm{kHz}$ transducer has been chosen with wide divergent angle and the couplant of silicon grease-based has been selected.

An array of 16-pairs of ultrasonic transducers with the ultrasonic transmitters and receivers, intermittently positioned, has been implemented. Based on Figure 9, it is found that the selected transducer not only has satisfied the characteristic needed for the important implementation of fan-shaped beam projection, but it is also noted that respectable voltage readings is measured evenly between the farthest distance of the sensor arrangement (Figure 5). The peak voltage readings for each different location are closely monitored so as to ensure proper mounting has been done and also to maximize the ultrasonic wave transmission. It is also important that the transducers vibrating element and pipe wall are parallel with each other. This has been solved prior to the sensor fixture that is designed with precision using $3 \mathrm{D}$ rapid prototyping to structure the sensors placing in a properly aligning manner.

The chosen operating frequency for the ultrasonic transducer for the ultrasonic transducer has clearly been balanced with the wide angle of reception and the size of the process column [15]. This is because complete attenuation did not occur since the ultrasound propagation has been successfully sensed by the corresponding receivers. The wide angle of reception for each transmitting source were able to cover up to 10 corresponding receivers (approximately $128^{\circ}$ ), which is important for the successful implementation of fan-beam projection technique.

\section{Acknowledgement}

The authors would like to thank the Ministry of Higher Education (MOHE) of Malaysia for supporting this work under MyBrain15 and Universiti Teknologi Malaysia's (UTM) support under Research University Fund (GUP - Grant H01).

\section{References}

[1] G. Falcone, G. F. Hewitt, C. 2009. Alimonti, Multiphase Flow Metering. Elsevier.

[2] D. Xie, W. Li, F. Wang, J. Zheng, Y. Zheng, G. Liang. 2007. A New Method for the Flowrate Measurement of Gas-Liquid Two-Phase Flow, IEEE Transactions on Instrumentation and Measurement. 56(4): 14951500

[3] B. S. Hoyle. 1996. Process Tomography Using Ultrasonic Sensors. Measurement Science and Technology. 7(3): 272.

[4] M. H. F. Rahiman. 2005. Non-invasive Imaging of Liquid/Gas Flow using Ultrasonic Transmission-mode Tomography. Master Dissertation.

[5] N. Wei-Nyap, Development of Ultrasonic Tomography for Composition Determination of Water and Oil Flow. 2005. Master Dissertation. Universiti Teknologi Malaysia.

[6] M. S. Saad. 2007. Concentration and Velocity Measurement of Flowing Objects using Optical and Ultrasonic Tomography. Master Dissertation. Universiti Teknologi Malaysia.

[7] N. M. Nor Ayob, S. Yaacob, Z. Zakaria, M. H. F. Rahiman, R. A. Rahim, M. R. Manan. 2010. Improving Gas Component Detection of an Ultrasonic Tomography System for Monitoring Liquid/Gas Flow. In: 6th 
International Colloquium on Signal Processing and Its Applications (CSPA 2010). 278-282.

[8] V. A. A.-O. Edward, L. Paul, Suzanne, M. Kresta. 2004. Handbook of Industrial Mixing. Science and Practice. John Wiley \& Sons, Inc.

[9] G. Steiner, F. Podd. 2006. A Non-Invasive And Non-Intrusive Ultrasonic Transducer Array For Process Tomography. In:XVIII IMEKO WORLD CONGRESS Metrology for a Sustainable Development. Rio de Janeiro, Brazil.

[10] R. A. Rahim, M. H. F. Rahiman, K. S. Chan, S. W. Nawawi. 2007. NonInvasive Imaging of Liquid/gas Flow Using Ultrasonic Transmissionmode Tomography. Sensors and Actuators A: Physical. 135(2): 337-345.

[11] M. H. F. Rahiman, R. A. Rahim, M. Tajjudin. 2006. Ultrasonic Transmission-Mode Tomography Imaging for Liquid/Gas Two-Phase Flow. Sensors Journal, IEEE. 6(6): 1706-1715.
[12] M. H. F. Rahiman, R. A. Rahim, N. M. N. Ayob. 2010. The Front-End Hardware Design Issue in Ultrasonic Tomography. Sensors Journal, IEEE. 10(7): 1276-1281.

[13] M. L. Sanderson, H. Yeung. 2002. Guidelines for the Use of Ultrasonic Non-Invasive Metering Techniques. Flow Measurement and Instrumentation. 13(4) 125-142.

[14] L. Schmerr, J. S. Song. 2007. Ultrasonic Nondestructive Evaluation Systems: Models and Measurements. Springer.

[15] N. M. Nor Ayob, M. H. F. Rahiman, Z. Zakaria, S. Yaacob, R. A. Rahim. 2010. Detection of Small Gas Bubble Using Ultrasonic TransmissionMode Tomography System. In: IEEE Symposium on Industrial Electronics \& Applications (ISIEA). 165-170.

[16] R. Abdul Rahim. S. Z. Mohd. Muji (January 2013). Optical Tomography: Image Improvement using Mix Projection of Parallel and Fan Beam Mode. Measurement Journal (ISSN: 0263-2241) Elsevier Science. 46: 19701978 
